\title{
Clinicopathological characteristics and survival outcomes for testicular choriocarcinoma: a population-based study
}

\author{
Huihuang $\mathrm{Li}^{1 \#}$, Zhiyong Cai ${ }^{1 \#}$, Renyu Liu ${ }^{2}$, Jiao $\mathrm{Hu}^{1}$, Jinbo Chen ${ }^{1}$, Xiongbing Z ${ }^{1}$ \\ ${ }^{1}$ Department of Urology, Xiangya Hospital, Central South University, Changsha, China; ${ }^{2}$ Department of Oncology, Xiangya Hospital, Central South \\ University, Changsha, China \\ Contributions: (I) Conception and design: X Zu, J Chen, H Li, Z Cai; (II) Administrative support: X Zu, J Chen; (III) Provision of study materials or \\ patients: H Li, Z Cai; (IV) Collection and assembly of data: R Liu, J Hu; (V) Data analysis and interpretation: H Li, Z Cai; (VI) Manuscript writing: \\ All authors; (VII) Final approval of manuscript: All authors. \\ \#These authors contributed equally to this work. \\ Correspondence to: Xiongbing Zu; Jinbo Chen. Department of Urology, Xiangya Hospital, Central South University, NO. 87 Xiangya Road, Changsha, \\ China. Email: zuxbxyyy@126.com; chenjinbo1989@yahoo.com.
}

Background: Due to the scarcity of cases of testicular choriocarcinoma (CC), its clinicopathological characteristics and prognosis have not been well summarized. Consequently, we conducted this populationbased case-control study to characterize the features of testicular CC.

Methods: The SEER database was used to extract qualified data. Dichotomous variables were compared by Pearson's Chi-squared or Fisher exact test. Survival variables were compared by Kaplan-Meier analyses and log-rank tests. The univariable and multivariable Cox regression analyses were applied to figure out risk factors for overall survival (OS) and cancer-specific survival (CSS). Propensity score matching (PSM) was used to control confounding factors in the study.

Results: In total, 788 patients with CC and 19,571 patients with seminoma were identified. Significant differences were found between two groups in terms of age ( $\leq 30$ years: $65.4 \%$ vs. $26.5 \%$; $>30$ years: $34.6 \%$ vs. $73.5 \%$; $\mathrm{P}<0.001)$, marital status $(28.8 \%$ vs. $52.1 \%$; $\mathrm{P}<0.001)$, laterality (proportion of bilateral tumors: $4.1 \%$ vs. $1.0 \%, \mathrm{P}<0.001$ ), tumors size ( $\leq 4 \mathrm{~cm}: 40.2 \%$ vs. $49.3 \% ;>4 \mathrm{~cm}: 45.8 \%$ vs. $43.0 \%$; $\mathrm{P}<0.001$ ), SEER stage (localized: $43.9 \%$ vs. $79.1 \%$; regional: $14.6 \%$ vs. $15.4 \%$; distant: $41.0 \%$ vs. $4.7 \%$; $\mathrm{P}<0.001$ ), surgery (92.4\% vs. 98.2\%; $\mathrm{P}<0.001)$ and chemotherapy (65.4\% vs. $19.8 \% ; \mathrm{P}<0.001)$. However, no differences were found between two groups after Propensity Score Matching (PSM). Furthermore, CC had worse outcomes than seminoma in terms of 5 -year rate of OS (85.5\% vs. $97.3 \%)$ and 5 -year rate of CSS (86.8\% vs. $98.6 \%)$. In univariable Cox hazard model, age, laterality, SEER stage (distant), surgery, chemotherapy and pathological type were independent prognostic factors for OS and CSS. However, in multivariable Cox hazard model, only age, SEER stage(distant) and surgery remained as the independent prognostic factor for OS and CSS.

Conclusions: Choriocarcinoma is exceedingly rare disease with metastases at initial diagnose and has poor survival even after treatment. Old age and advanced tumor stage indicate a poor prognosis, while surgery therapy can improve prognosis. Nevertheless, longer-term studies with larger population of patients are needed to verify their biological behavior and therapeutic efficacy.

Keywords: Testicular choriocarcinoma; non-seminoma; clinicopathological characteristics; survival time

Submitted Jul 04, 2020. Accepted for publication Nov 15, 2020.

doi: 10.21037/tau-20-1061

View this article at: http://dx.doi.org/10.21037/tau-20-1061 


\section{Introduction}

In 2020, it is estimated that about 9610 new cases with testicular tumors will occur in the United States (1). Germ cell tumors (GCT) occupy $95 \%$ of testicular tumors, which can be divided into two histologic categories: seminoma and non-seminoma (2). Choriocarcinoma (CC) is one of the subtypes of non-seminoma, which only accounts for $7 \%$ to $8 \%$ of testicular tumors (3). Although the incidence is not high, it is the most common solid tumor in men between 20 to 39 years old, and the median age of patients suffering from testicular choriocarcinoma is only 29 years old (4). Moreover, unlike other GCT, it's very aggressive and spread widely via hematogenous route, consequently with poor prognosis. In aspect of therapy, the primary treatment is radical inguinal orchiectomy, other various therapeutic regimens including retroperitoneal lymph node dissection (RPLND), chemotherapy and surveillance are applied according to patient's pathological report, disease histology, stage and risk classification $(5,6)$.

Due to the scarcity of cases and the complexity of the disease, the clinical features and prognostic factors of testicular choriocarcinoma are still not well characterized. Thus, we searched the Surveillance, Epidemiology, and End Results (SEER) database and summarized clinicopathological characteristics and survival outcomes for testicular choriocarcinoma in this study. Because seminoma is the most common malignant tumor among testicular tumors, we set it as a control group to show the clinical features of choriocarcinoma.

We present the following article in accordance with the STROBE reporting checklist (available at http://dx.doi. org/10.21037/tau-20-1061).

\section{Methods}

\section{Data extraction}

The SEER database of the National Cancer Institute collects cancer diagnosis, treatment, and survival data for approximately $30 \%$ of the US population. SEER*Stat software (version 8.3.6) was used for extracting data from "Incidence-SEER 18 Regs Custom Data (with additional treatment fields), Nov 2018 Sub (1975-2016 varying)" database.

The patients who diagnosed as testicular choriocarcinoma and seminoma from 1998 to 2016 based on the third edition of the International Classification of Diseases for Oncology (ICD-O-3) were included in this study. Histology codes for each disorder were following: 9100/3 (Choriocarcinoma), 9101/3 (Choriocarcinoma combined with other germ cell elements) and 9061/3 (Seminoma). In the SEER database, Surgery therapy of testicular malignancies include local or partial excision of testicle, excision of testicle with or without cord and radical orchiectomy.

There are three main principals to filter the cases. Firstly, patients included in our study not only need be confirmed their malignant status by histology, but also their primary tumor limited to one site. Secondly, the cases lack of detailed follow-up information and cause of death were ruled out. Last but not the least, as radiotherapy is not recommended for patients with non-seminoma, in order to prevent selection bias, patients underwent radiation treatment were excluded from the cohort. Detailed screening process were shown in the flow diagram of Figure 1.

The study was conducted in accordance with the Declaration of Helsinki (as revised in 2013). Because the original data came from the SEER database, the study didn't need to approved by ethics board of institution and the participants didn't need to give informed consent before taking part.

\section{Variables}

The following SEER variables were selected for our study: age, marital status, laterality, tumor size, SEER stage, therapeutic methods (including: surgery and chemotherapy). Age was divided into two groups: $\leq 30$ and $>30$ years; tumor size was categorized into two groups: $\leq 4$ and $>4 \mathrm{~cm}$; SEER stages were classified into three groups by combination of the most precise clinical and pathological documentation recorded in the SEER program, which were localized, regional and distant. Localized meant "within organ", regional defined by "extension to adjacent organs or to regional lymph nodes" and distant determined by "direct extension or metastasis". Surgery was classified into "Yes", "No" and "Unknown". Chemotherapy was classified into "No/Unknown" and "Yes". The primary survival outcomes were overall survival (OS) which calculated from diagnosis to any cause of death and cancer-specific survival (CSS) which calculated from diagnosis to death caused by testis carcinoma.

\section{Statistical analysis}

All the data were analyzed by R language (v4.0.0) software, 

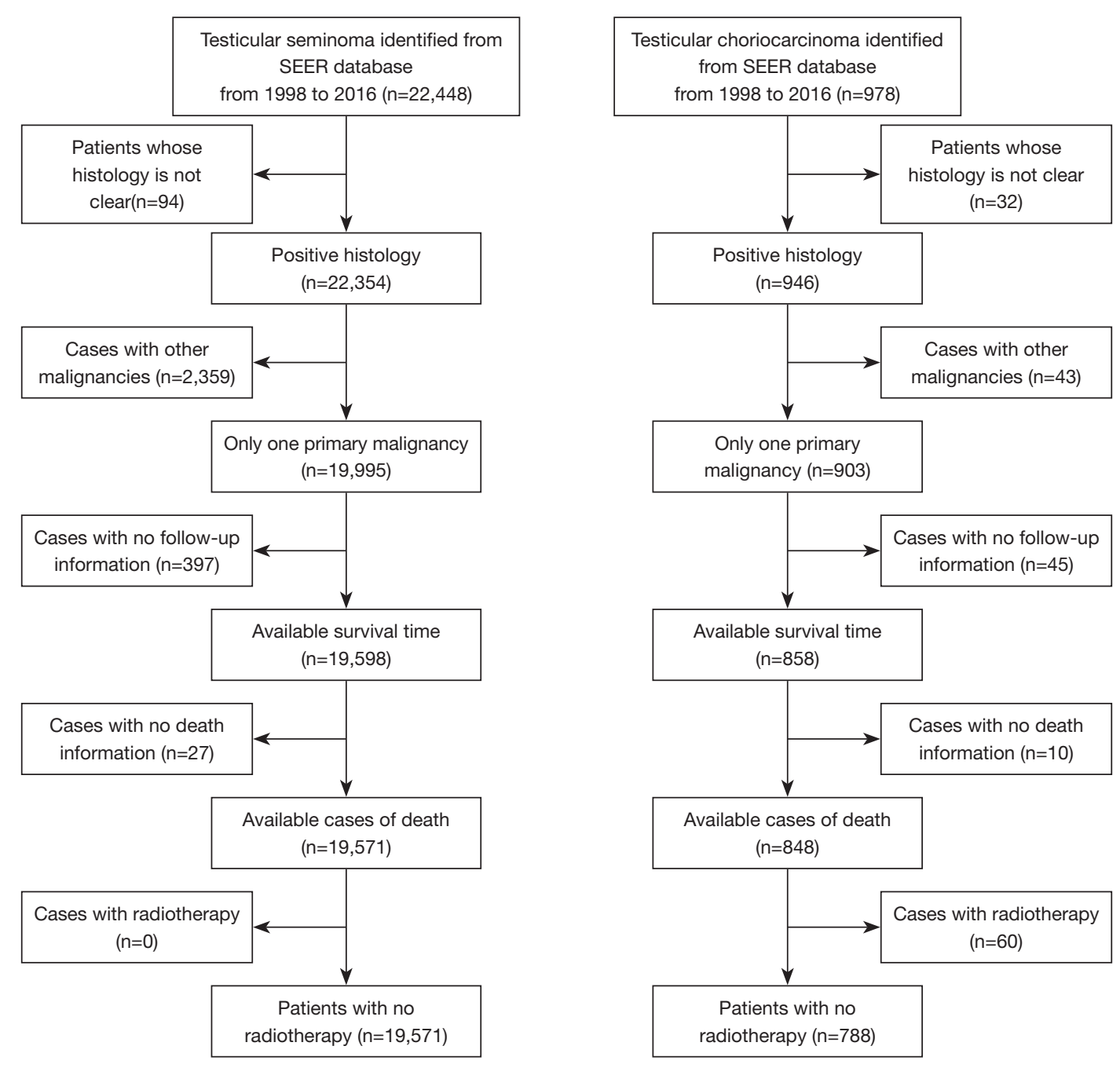

Figure 1 Flow diagram for patients' selection.

and the elementary packages were "gmodels", "nonrandom", "survival" and "survminer". Clinicopathological characteristics for testicular choriocarcinoma and seminoma were analyzed by using Chi-squared test or Fisher's exact test. Propensity score matching was applied to process the analysis with a $1: 2$ ratio of choriocarcinoma to seminoma, for balancing the confounding bias in the study. KaplanMeier survival curves were used to show median survival time, and log-rank tests were used to determine the significance. The univariable and multivariable Cox proportional hazards models were used to assess the impact of related risk factors on OS and CSS. Two-sided tests were used and $\mathrm{P}<0.05$ was deemed as statistically significant.

\section{Results}

\section{Clinicopathological characteristics of testicular choriocarcinoma patients and seminoma patients}

In total, 788 patients with choriocarcinoma and 19,571 patients with seminoma were identified in the SEER database. As shown in Table 1, In comparison with the patients in seminoma group, patients in the choriocarcinoma group had larger proportion of young people $(\leq 30$ years: $65.4 \%$ vs. $26.5 \%$; $>30$ years: $34.6 \%$ vs. $73.5 \%$; $\mathrm{P}<0.001)$, smaller proportion of getting married ( $28.8 \%$ vs. $52.1 \%$; $\mathrm{P}<0.001)$, larger proportion of bilateral tumors $(4.1 \% v s$. $1.0 \%, \mathrm{P}<0.001)$, larger proportion of big tumors $(\leq 4 \mathrm{~cm}$ : 
Table 1 Clinicopathological characteristics of patients with testicular choriocarcinoma (CC) or seminoma

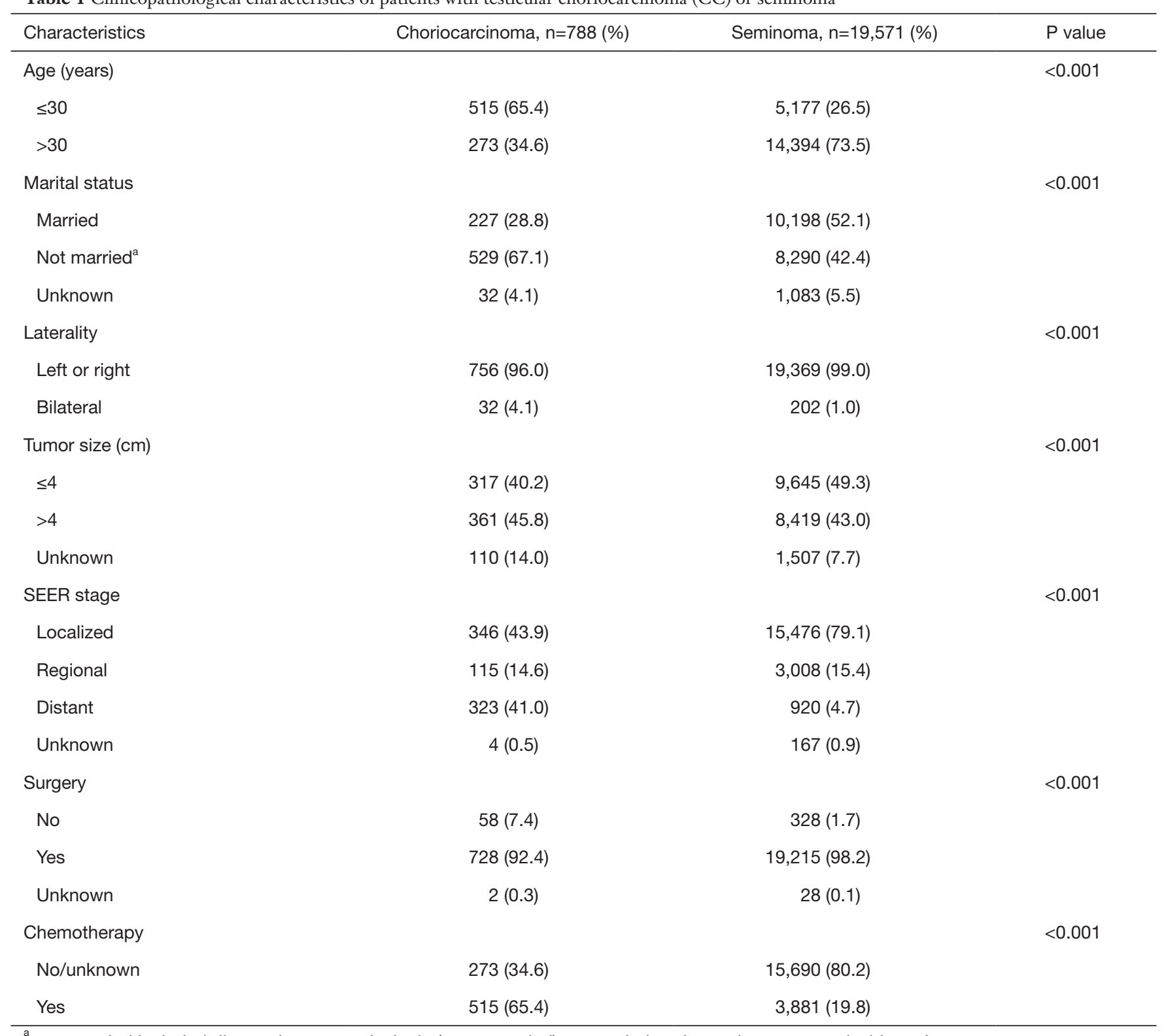

${ }^{a}$, not married included divorced, separated, single (never married), unmarried or domestic partner and widowed.

40.2\% vs. $49.3 \%$; $>4$ cm: $45.8 \%$ vs. $43.0 \%$; $\mathrm{P}<0.001)$, larger proportion of advanced SEER stage (localized: $43.9 \%$ vs. $79.1 \%$; regional: $14.6 \%$ vs. $15.4 \%$; distant: $41.0 \%$ vs. $4.7 \%$; $\mathrm{P}<0.001)$, smaller proportion of patients receiving surgery (92.4\% vs. 98.2\%; $\mathrm{P}<0.001)$ and larger population accepted chemotherapy $(65.4 \%$ vs. $19.8 \% ; \mathrm{P}<0.001)$.

Due to the imbalance of variables between two groups, selection bias and confounding variables probably affected the results in the end. Consequently, in order to bring the result close to the effects of randomized controlled studies, we applied propensity score matching (PSM) to balance the confounding bias in the study. After PSM, no significant differences were found between two groups (Table S1).

\section{Survival rates of testicular choriocarcinoma patients and seminoma patients}

As shown in Figure 2, the choriocarcinoma group had worse CSS and OS than the seminoma group $(\mathrm{P}<0.0001)$. We used 5-year survival rates instead of median survival time 

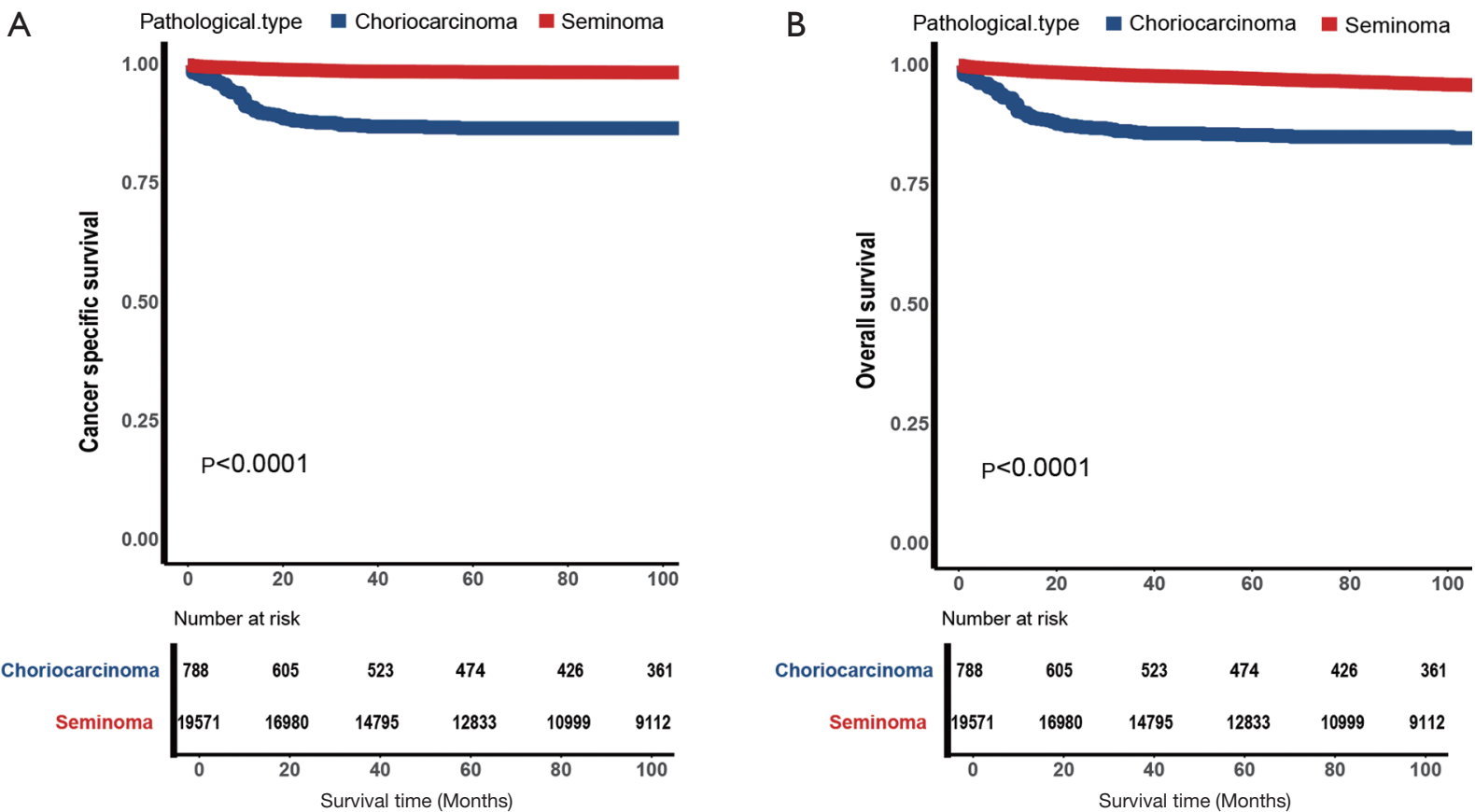

Figure 2 Kaplan-Meier estimate for patients with testicular choriocarcinoma or seminoma. (A) Cancer-specific survival (CSS) for all patients with testicular choriocarcinoma (CC) or seminoma; (B) overall survival (OS) for all patients with testicular CC or seminoma.

in the study and found that survival outcomes in the SEER database in both groups were over 50 percent, the 5 -year rate of CSS were $86.8 \%$ versus $98.6 \%$ and 5 -year rate of OS were $85.5 \%$ versus $97.3 \%$, in the choriocarcinoma group and seminoma group respectively. As shown in Figure 3, CSS and OS after PSM, the choriocarcinoma group still had worse outcomes than that in the seminoma group $(\mathrm{P}<0.0001), 5$-year rate of CSS were $90.0 \%$ versus $95.2 \%$ and 5 -year rate of OS were $89.0 \%$ versus $94.1 \%$, in the choriocarcinoma group and seminoma group respectively.

\section{Prognostic factors for OS and CSS in testicular choriocarcinoma patients}

In univariate cox proportional hazards regression model, we found that age $\geq 30$, bilateral tumors, SEER stage in distant and chemotherapy were negative prognostic factors for testicular choriocarcinoma patients in terms of OS and CSS $(\mathrm{P}<0.05)$. Surgery and mixed component of tumor pathology were positive prognostic factors for better OS and CSS $(\mathrm{P}<0.05)$ (Table S2). Further, we applied multivariate cox proportional hazards regression model to validate the results, found that age $\geq 30$ and SEER stage in distant were still negative prognostic factors in terms of OS and CSS, surgery was sole positive factor for better OS and CSS (Table 2).

\section{Discussion}

Although testicular cancer is uncommon which only accounts for less than $1 \%$ of all male tumors (7), the incidence of testicular cancer has been rising in the past 20 years (8). As a part of GCT, choriocarcinoma is a rare pathological type, whose pure form only accounts for approximately $0.5 \%$ of all testis tumors (9). Due to scarcity of cases, most of articles about it are case reports, hence, the clinicopathological features of the disease have not been well summarized so far. We collected 788 patients with choriocarcinoma and 19,571 patients with seminoma by searching SEER database. In our study, the patients in the choriocarcinoma group were younger, the ratio of bilateral tumors was higher, the size of tumors was larger, the SEER stage was more advanced, the ratio of receiving surgery was lower, the ratio of accepting chemotherapy was higher. Alvarado-Cabrero et al. published a series study on pure CC and predominant CC in GCT of the testis, 15 patients were included (10) with median age of 29 years old, the mean size of tumor was $6.5 \mathrm{~cm}$, all of them presented 
A
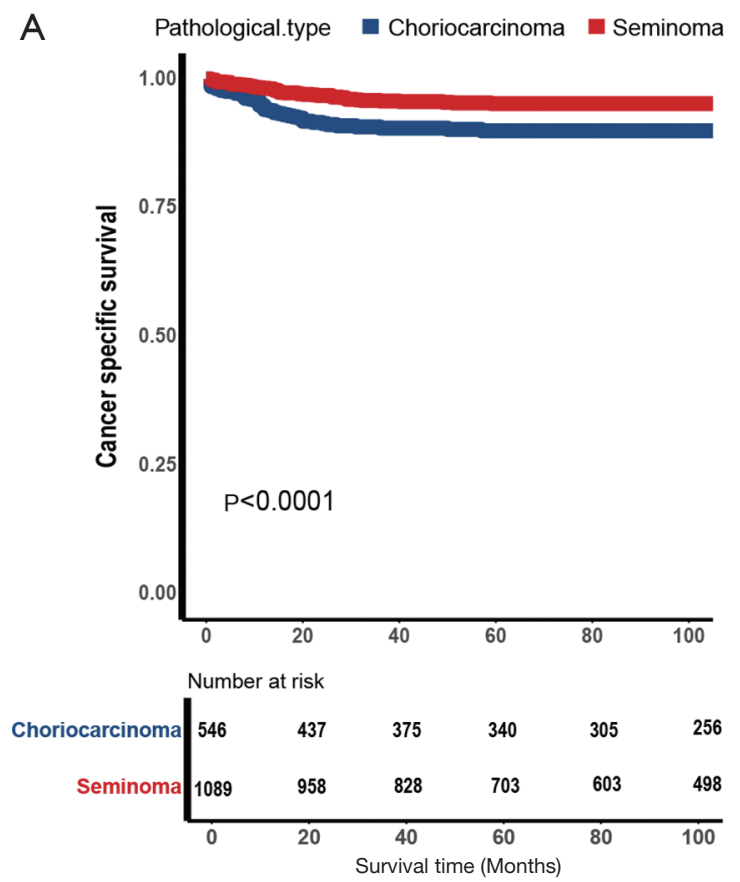

B
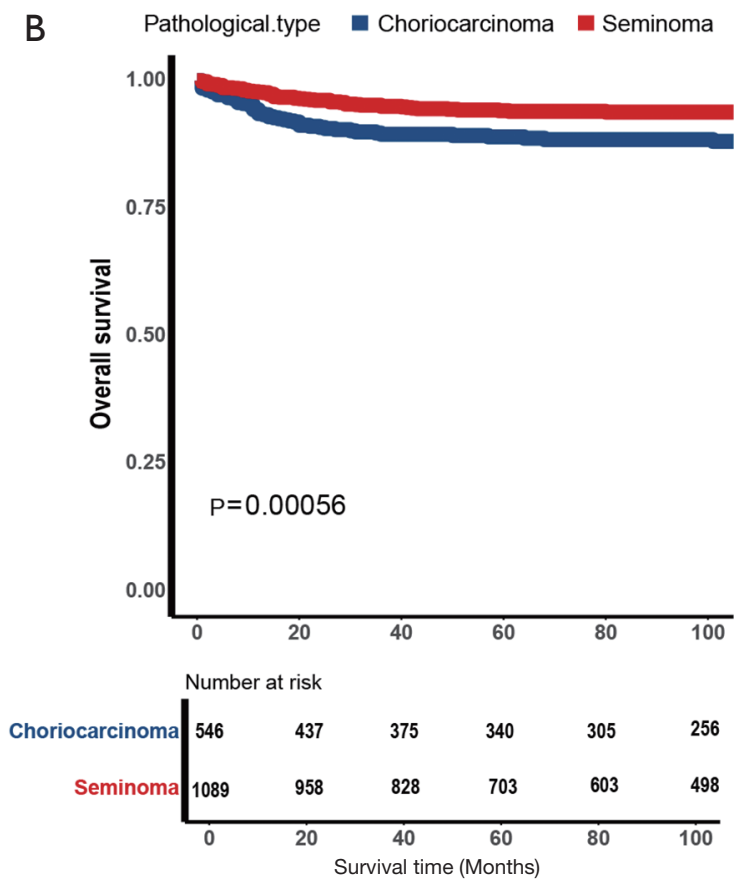

Figure 3 Kaplan-Meier estimate for patients with testicular choriocarcinoma or seminoma. (A) Cancer-specific survival (CSS) after propensity score matching (PSM) for matched patients with testicular choriocarcinoma (CC) or seminoma; (B) overall survival (OS) after PSM for matched patients with testicular CC or seminoma.

with unilateral tumors and metastasized to distant sites. The age, SEER stage and tumor size were similar to our study, these features are indicators for worse prognosis of choriocarcinoma. The difference in laterality might be caused by limited sample size in Isabel's study.

In our results, Distant metastasis accounted for $41.0 \%$ in all patients. High metastasis rate is a major feature of CC. The ability of invading to host vessels make CC an aggressive cancer which lead to a poor prognosis. Malek et al. suggested that chromosomal anomalies such as gaining or losing part of chromosome, e.g., i (12p) (11) might be the cause of early invasion of the tumor. Moreover, several studies indicated that vasculogenic mimicry, epithelial-tomesenchymal transition and $\mathrm{G}$ protein-coupled receptor 1 expression may also contribute to the character of early vessel invasion $(12,13)$.

In the survival outcomes, we found that 5 -year rate of CSS were $86.8 \%$ in CC and 5-year rate of OS in CC were $85.5 \%$, which were worse than that of seminoma patients. Stang et al. have done a research on different types of GCT in testis, and reported that 5-year relative survival for seminoma was close to $97.6 \%$, non-seminoma was $93.3 \%$, choriocarcinoma was the lowest, about $80.1 \%$ (14). Their study also validated the fact that the prognosis of $\mathrm{CC}$ is worse than that of seminoma. In addition, the results of our multivariable cox proportional hazards model suggested that age $\geq 30$ and SEER stage in distant were adverse predictors for OS and CSS. Surgery was positive predictor for OS and CSS. Jiang et al. revealed that patients with 34 years old and younger had significantly longer OS (15). Stephenson et al. reported that by excluding clinical stage IIb non-seminoma patients from testicular tumor, the 4-year progressionfree survival rate improved from $83 \%$ to $96 \%(16)$. which in agreement with our results that patients in younger age and localized tumor stage have better prognosis. Based on symptoms, physical examinations, imaging examinations on testes and serum tumor markers, all suspicious patients are recommended to have radical inguinal orchiectomy or inguinal exploration (2). Liu et al. analyzed various factors for prognosis in non-gestational ovarian choriocarcinoma patients and found that ovariectomy is a good prognostic factor for OS (17). Once choriocarcinoma is confirmed, subsequent treatment regimens such as: retroperitoneal lymph node dissection (RPLND), chemotherapy and surveillance are employed individually or in combination according to TMN stage, $\mathrm{S}$ stage and clinical risk factors. 
Table 2 Multivariate Cox proportional hazards regression model analysis of overall survival and cancer-special survival for testicular choriocarcinoma (CC) patients

\begin{tabular}{|c|c|c|c|c|}
\hline Characteristics & \multicolumn{2}{|c|}{ Cancer-special survival } & \multicolumn{2}{|c|}{ Overall survival } \\
\hline \multicolumn{5}{|l|}{ Age } \\
\hline$\leq 30$ years & Reference & - & Reference & - \\
\hline$>30$ years & $1.593(1.066-2.380)$ & 0.023 & $1.600(1.100-2.326)$ & 0.014 \\
\hline Left or right & Reference & - & Reference & - \\
\hline Bilateral & $1.256(0.640-2.464)$ & 0.508 & $1.161(0.599-2.248)$ & 0.659 \\
\hline \multicolumn{5}{|l|}{ SEER stage } \\
\hline Localized & Reference & - & Reference & - \\
\hline \multicolumn{5}{|l|}{ Surgery } \\
\hline No & Reference & - & Reference & - \\
\hline Yes & $0.429(0.241-0.764)$ & 0.004 & $0.476(0.274-0.826)$ & 0.008 \\
\hline \multicolumn{5}{|l|}{ Chemotherapy } \\
\hline None/unknown & Reference & - & Reference & - \\
\hline Yes & $0.874(0.395-1.934)$ & 0.739 & $0.932(0.453-1.919)$ & 0.849 \\
\hline \multicolumn{5}{|l|}{ Pathological type } \\
\hline Pure choriocarcinoma & Reference & - & Reference & - \\
\hline
\end{tabular}

${ }^{a}$, not married included divorced, separated, single (never married), unmarried or domestic partner and widowed.

Mazzone et al. compared RPLND to no RPLND after primary treatment of stage I non-seminoma of testis, found that RPLND was associated with lower cancer-specific mortality (18). In our study, surgery is also regarded as a positive factor for patients' prognosis. However, with the limitation of SEER database, not only RPLND wasn't included in the surgery therapy, but also lack of specific analysis on different surgical methods (e.g., local or partial excision of testicle, excision of testicle with or without cord and radical orchiectomy). Consequently, more concrete studies are needed to verify our conclusion.

Although chemotherapy was not associated with better CSS and OS in our study, we still consider chemotherapy is beneficial for patient's survival time. Tandstad et al. conducted a multicenter study on 745 patients with clinical stage I non-seminoma and showed that $41.7 \%$ of vascular invasion patients relapsed in surveillance group, but only $3.2 \%$ of vascular invasion patients relapsed in BEP chemotherapy group (19) over period of time. Oliver et al. studied survival events after adjuvant chemotherapy for Stage I non-seminoma and found that the recurrence rate of high-risk patients who accepted adjuvant treatment decreased by $24 \%$, however, those who accepted active surveillance their recurrence rate declined only by $11 \%$ (20). Both of them demonstrated that chemotherapy is indispensable in the localized CC patients. In addition, Stephenson et al. found that with increasing patients received chemotherapy over time, their relapse-free survival were significantly improved from $84 \%$ to $98 \%$ for clinical stage IIA and IIB nonseminomatous germ cell testicular cancer (21).

NCCN guideline recommends radiotherapy to 
seminoma at stage Ia-IIb, but to non-seminoma, no specific instruction is stated on this regard. Feldman et al. have done a research on brain metastases from GCT and found out that patients with radiotherapy couldn't change the course of tumor development (22). Doyle et al. studied the effect of whole brain radiation treatment on GCT patients and found that RT was not innocuous and could cause severe CNS toxicity (23). But no agreements were reached on this matter in the field. Casey et al. reported that patients suffered from non-seminomatous germ cell tumor (NSGCT) brain metastasis had a longer 4-year OS after radiotherapy (24). Further studies evaluating radiation therapy on NSGCT is in great need to clarify its efficacy.

There are several limitations in the study. First of all, there are selective bias and other confounding factors in a retrospective study. Next, data of chemotherapy coming from SEER database have inevitable bias. Last but not the least, comparison of efficacy between different treatment regimens may be affected by other supplementary therapeutic method applied in the process.

\section{Conclusions}

Choriocarcinoma is exceedingly rare disease with metastases at initial diagnose and has poor survival even after treatment. Old age and advanced tumor stage indicate a poor prognosis, while surgery therapy can improve prognosis. Nevertheless, longer-term studies with larger population of patients are needed to verify their biological behavior and therapeutic efficacy.

\section{Acknowledgments}

We sincerely thank You'e He for revising the language of this article.

Funding: This study was supported by the National Natural Science Foundation of China (81873626, 81902592), Hunan Province Key R\&D Program (2019SK2202) and Xiangya Hospital Youth Fund (2018Q09).

\section{Footnote}

Reporting Checklist: The authors have completed the STROBE reporting checklist. Available at http://dx.doi. org/10.21037/tau-20-1061

Conflicts of Interest: All authors have completed the ICMJE uniform disclosure form (available at http://dx.doi. org/10.21037/tau-20-1061). XZ serves as an unpaid editorial board member of Translational Andrology and Urology from Mar 2019 to Feb 2021. The other authors have no conflicts of interest to declare.

Ethical Statement: The authors are accountable for all aspects of the work in ensuring that questions related to the accuracy or integrity of any part of the work are appropriately investigated and resolved. The study was conducted in accordance with the Declaration of Helsinki (as revised in 2013). Because the original data came from the SEER database, the study didn't need to approved by ethics board of institution and the participants didn't need to give informed consent before taking part.

Open Access Statement: This is an Open Access article distributed in accordance with the Creative Commons Attribution-NonCommercial-NoDerivs 4.0 International License (CC BY-NC-ND 4.0), which permits the noncommercial replication and distribution of the article with the strict proviso that no changes or edits are made and the original work is properly cited (including links to both the formal publication through the relevant DOI and the license). See: https://creativecommons.org/licenses/by-nc-nd/4.0/.

\section{References}

1. Siegel RL, Miller KD, Jemal A. Cancer statistics, 2020. CA Cancer J Clin 2020;70:7-30.

2. Gilligan T, Lin DW, Aggarwal R, et al. Testicular Cancer, Version 2.2020, NCCN Clinical Practice Guidelines in Oncology. J Natl Compr Canc Netw 2019;17:1529-54.

3. Humphrey PA. Choriocarcinoma of the testis. J Urol 2014;192:934-5.

4. Hassan O, Epstein JI. The Clinical Significance of a Small Component of Choriocarcinoma in Testicular Mixed Germ Cell Tumor (MGCT). Am J Surg Pathol 2018;42:1113-20.

5. Vasdev N, Moon A, Thorpe AC. Classification, epidemiology and therapies for testicular germ cell tumours. Int J Dev Biol 2013;57:133-9.

6. Sarıcı H, Telli O, Eroğlu M. Bilateral testicular germ cell tumors. Turk J Urol 2013;39:249-52.

7. Cheng L, Albers P, Berney DM, et al. Testicular cancer. Nat Rev Dis Primers 2018;4:29.

8. Ghazarian AA, Kelly SP, Altekruse SF, et al. Future of testicular germ cell tumor incidence in the United States: Forecast through 2026. Cancer 2017;123:2320-8. 
9. Reilley MJ, Pagliaro LC. Testicular choriocarcinoma: a rare variant that requires a unique treatment approach. Curr Oncol Rep 2015;17:2.

10. Alvarado-Cabrero I, Hernandez-Toriz N, Paner GP. Clinicopathologic analysis of choriocarcinoma as a pure or predominant component of germ cell tumor of the testis. Am J Surg Pathol 2014;38:111-8.

11. Malek NP, Casper J, Looijenga LH, et al. Quantification of additional short arms of chromosome 12 in germ cell tumours using the polymerase chain reaction. Eur J Cancer 1997;33:1488-94.

12. Xue Y, Sun R, Zheng W, et al. Forskolin promotes vasculogenic mimicry and invasion via Notch-1-activated epithelial-to-mesenchymal transition in syncytiolization of trophoblast cells in choriocarcinoma. Int J Oncol 2020;56:1129-39.

13. Huang B, Zhu W, Chang J, et al. Role of $\mathrm{G}$ proteincoupled receptor 1 in choriocarcinoma progression. Am J Physiol Cell Physiol 2019;317:C556-65.

14. Stang A, Jansen L, Trabert B, et al. Survival after a diagnosis of testicular germ cell cancers in Germany and the United States, 2002-2006: a high resolution study by histology and age. Cancer Epidemiol 2013;37:492-7.

15. Jiang F, Xiang Y, Feng FZ, et al. Clinical analysis of 13 males with primary choriocarcinoma and review of the literature. Onco Targets Ther 2014;7:1135-41.

16. Stephenson AJ, Bosl GJ, Motzer RJ, et al. Retroperitoneal lymph node dissection for nonseminomatous germ cell testicular cancer: impact of patient selection factors on outcome. J Clin Oncol 2005;23:2781-8.

17. Liu X, Zhang X, Pang Y, et al. Clinicopathological factors and prognosis analysis of 39 cases of nongestational ovarian choriocarcinoma. Arch Gynecol Obstet
2020;301:901-12.

18. Mazzone E, Mistretta FA, Knipper S, et al. Contemporary Assessment of Long-Term Survival Rates in Patients With Stage I Nonseminoma Germ-Cell Tumor of the Testis: Population-Based Comparison Between Surveillance and Active Treatment After Initial Orchiectomy. Clin Genitourin Cancer 2019;17:e1153-62.

19. Tandstad T, Ståhl O, Håkansson U, et al. One course of adjuvant BEP in clinical stage I nonseminoma mature and expanded results from the SWENOTECA group. Ann Oncol 2014;25:2167-72.

20. Oliver RT, Ong J, Shamash J, et al. Long-term follow-up of Anglian Germ Cell Cancer Group surveillance versus patients with Stage 1 nonseminoma treated with adjuvant chemotherapy. Urology 2004;63:556-61.

21. Stephenson AJ, Bosl GJ, Motzer RJ, et al. Nonrandomized comparison of primary chemotherapy and retroperitoneal lymph node dissection for clinical stage IIA and IIB nonseminomatous germ cell testicular cancer. J Clin Oncol 2007;25:5597-602.

22. Feldman DR, Lorch A, Kramar A, et al. Brain Metastases in Patients With Germ Cell Tumors: Prognostic Factors and Treatment Options--An Analysis From the Global Germ Cell Cancer Group. J Clin Oncol 2016;34:345-51.

23. Doyle DM, Einhorn LH. Delayed effects of whole brain radiotherapy in germ cell tumor patients with central nervous system metastases. Int J Radiat Oncol Biol Phys 2008;70:1361-4.

24. Casey DL, Pitter KL, Imber BS, et al. High-dose radiation therapy is needed for intracranial control and long-term survival in patients with non-seminomatous germ cell tumor brain metastases. J Neurooncol 2019;142:523-8.
Cite this article as: $\mathrm{Li} \mathrm{H}$, Cai Z, Liu R, Hu J, Chen J, Zu X. Clinicopathological characteristics and survival outcomes for testicular choriocarcinoma: a population-based study. Transl Androl Urol 2021;10(1):408-416. doi: 10.21037/tau-20-1061 


\section{Supplementary}

Table S1 Clinicopathological characteristics of patients with testicular choriocarcinoma (CC) or seminoma after PSM

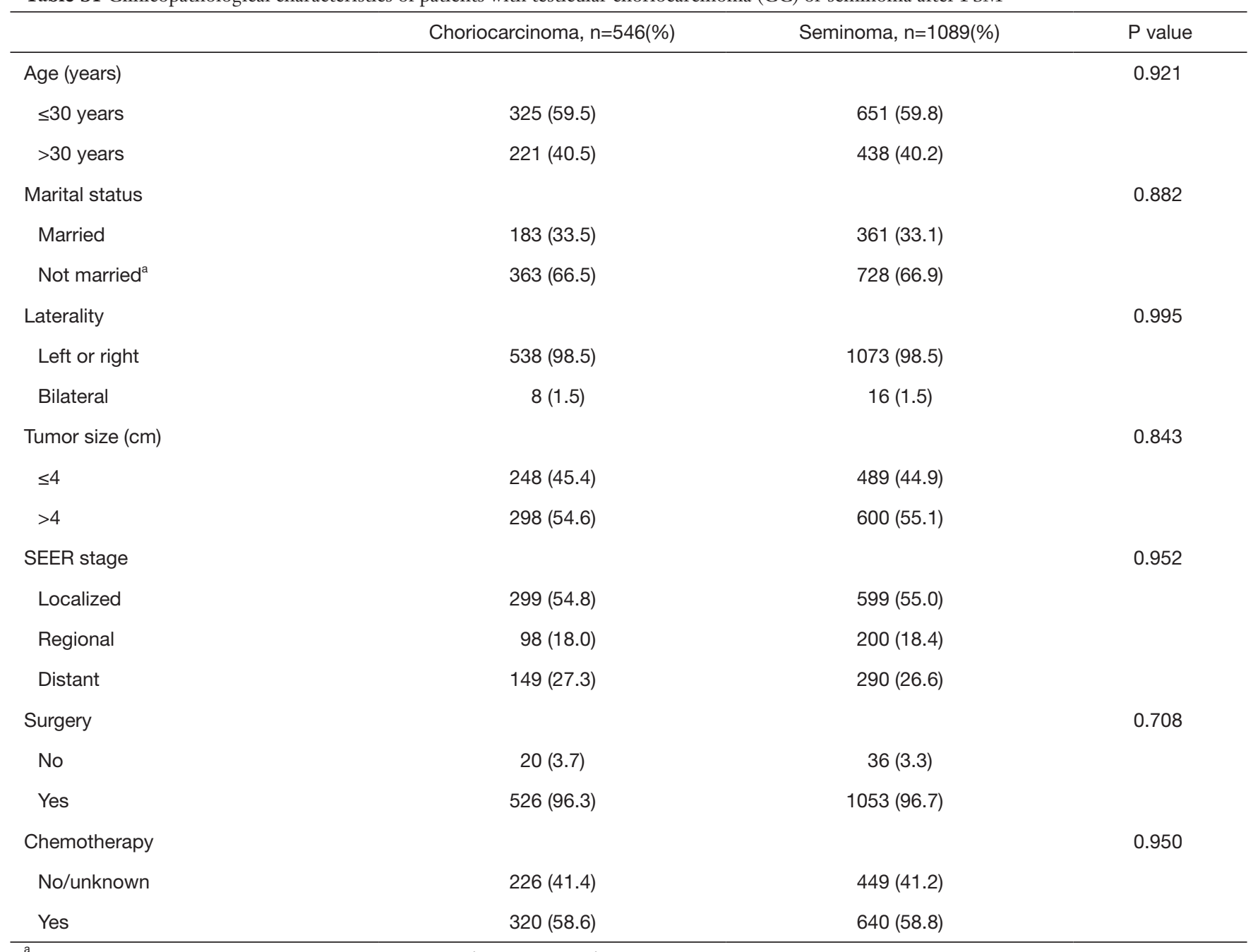

${ }^{\mathrm{a}}$, not married included divorced, separated, single (never married), unmarried or domestic partner and widowed. 
Table S2 Univariate Cox proportional hazards regression model analysis of overall survival and cancer-special survival for testicular choriocarcinoma (CC) patients

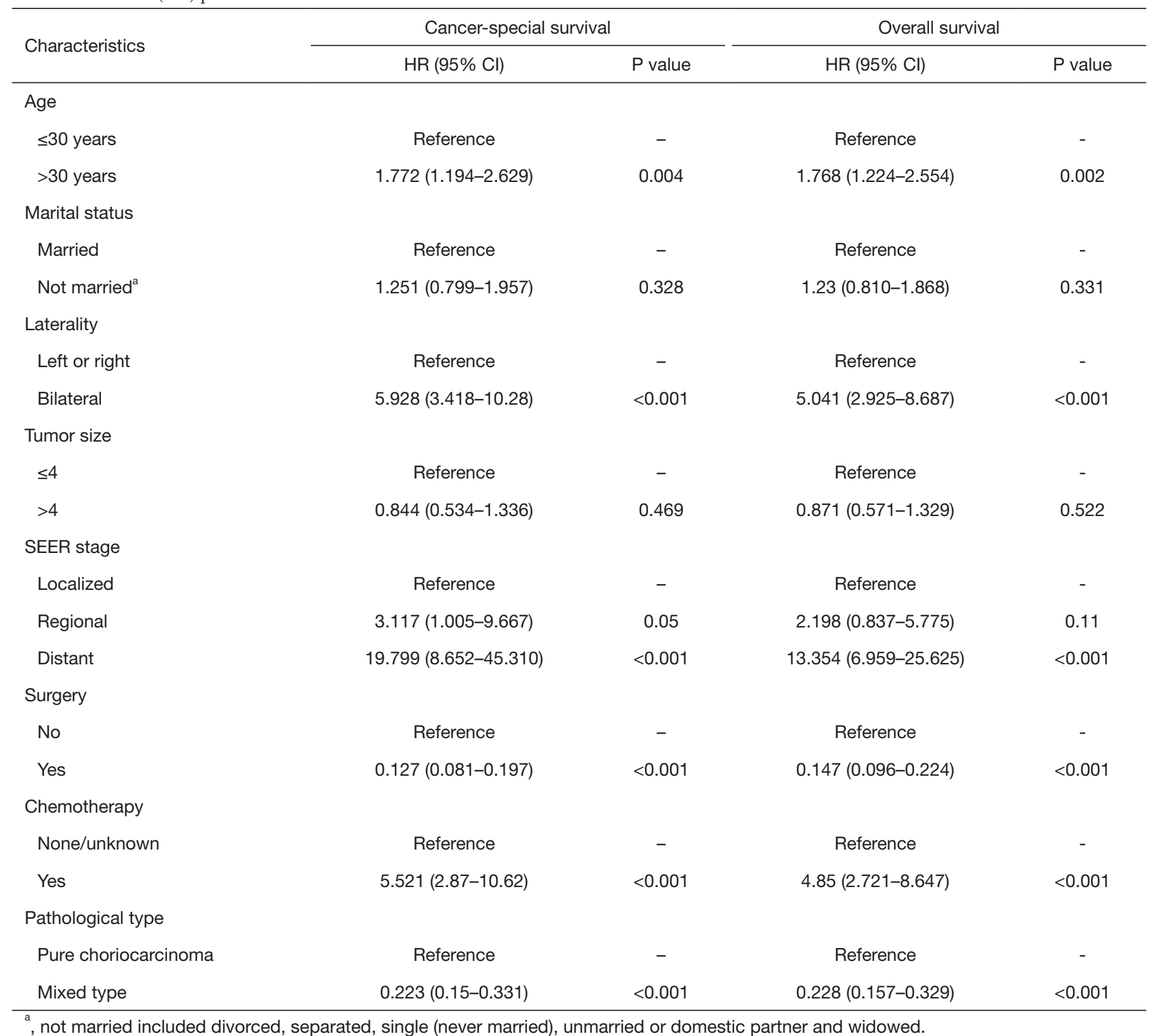

\footnotetext{
${ }^{\mathrm{a}}$, not married included divorced, separated, single (never married), unmarried or domestic partner and widowed.
} 